\title{
The Erlangen Score Algorithm in the diagnosis and prediction of the progression from subjective cognitive decline and mild cognitive impairment to Alzheimer-type dementia
}

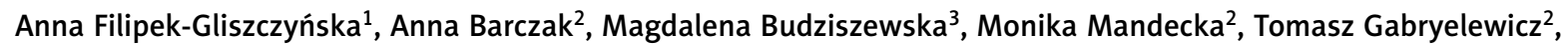 \\ Maria Barcikowska² \\ ${ }^{1}$ Neurology Clinic MSWiA Hospital, Warsaw, ${ }^{2}$ Department of Neurodegenerative Disorders, Mossakowski Medical Research Centre, \\ Polish Academy of Science, Warsaw, ${ }^{3}$ Faculty of Psychology, University of Warsaw, Warsaw, Poland
}

\begin{abstract}
The evaluation of cerebrospinal fluid (CSF) biomarkers for Alzheimer's disease (AD) ( $\beta$-amyloid, $t$-tau, $p$-tau) can be used to estimate the risk of developing dementia in patients at the pre-clinical stages of $A D$, i.e. subjective cognitive decline $(S C D)$ and mild cognitive impairment $(M C I)$. Erlangen Score Algorithm allows interpretation of CSF biomarker concentrations and is cut-off value independent. The aim of this study was to establish if this algorithm can be applied for routine diagnostic testing in clinical and preclinical subjects and has prognostic value. We analysed 217 patients from the memory clinic with the diagnosis of SCD $(n=31), \mathrm{MCl}(n=104)$, and $A D(n=82)$ with clinical follow-up amounting to 14.33 months $(S D=6.82)$. It was found that the highest Erlangen Score dominated in the $A D$ group and was the rarest in the $S C D$ group. In the group of patients with progression of symptoms during our period of observation, the AD pathology was confirmed in $93.75 \%$ of cases. Among the non-progressing subjects $(n=119)$ the algorithm indicated the risk of developing $A D$ as possible in $40.34 \%$ and probable in $15.97 \%$ of cases. To conclude, the Erlangen Score Algorithm is a useful tool to determinate the risk of developing AD before the onset of dementia or to confirm the $A D$ diagnosis. It is extremely valuable in preclinical stages of $A D$ for planning purposes and early intervention as well as for future clinical trials.
\end{abstract}

Key words: Alzheimer's disease, mild cognitive impairment, subjective cognitive decline, cerebrospinal fluid biomarkers, Erlangen Score Algorithm.

\section{Introduction}

The sequence of pathophysiological changes in the Alzheimer's process (extracellular aggregation of $\beta$-amyloid plaques and neurofibrillary degeneration caused by the intracellular concentration of hyperphosphorylated tau protein) starts many years before the full symptoms of the disease manifest themselves $[6,22]$. The $A \beta_{1-42}$ peptide is especially the one most prone to an aggregation resulting in amyloid plaques, which are a neuropathological hallmark of Alzheimer's disease (AD), along with the formation of neurofibrillary tangles (NFTs) [17]. The continuum of events leading to the develop- 
ment of the full-blown manifestation of $A D$ is of key importance, and it is a starting point for dividing $A D$ as a disease into several stages: the pre-clinical stage of the disease, i.e. subjective cognitive decline (SCD), mild cognitive impairment $(\mathrm{MCl})$, and the actual dementia stage.

Subjective cognitive decline is characterised by the presence of subjective complaints on cognitive worsening, but which are not detected in objective evaluation, even in the neuropsychological assess ment. Patients with $\mathrm{MCl}$ also report problems with cognition, but in this case the worsening is noticeable by informants and confirmed in neuropsychological assessment. Nevertheless, those patients remain independent in complex daily activities. More pronounced cognitive worsening along with the presence of problems in conducting everyday tasks are hallmarks of dementia. The presence of complaints of cognitive difficulties, regardless of their objectiveness, differentiate those stages of $A D$ from normal controls. To identify patients at the pre-clinical stages of Alzheimer's disease, i.e. SCD and $\mathrm{MCl}$, it is crucial to mark biological indicators of the earliest pathophysiological process of Alzheimer's disease.

The literature presents a great deal of evidence that patients with $A D$ tend to have lower $A \beta_{1-42}$ values and higher t-tau (total tau) and p-tau (tau phosphorylated at threonine 181) proteins in the cerebrospinal fluid (CSF) tests $[3,20,21,31]$.

The evaluation of lowered levels of $A \beta_{1-42}$ tends to display sensitivity of $86 \%$ and specificity of $89 \%$ in patients with $A D$ against the healthy population. The evaluation of t-tau in CSF reveals a sensitivity of $80 \%$ and specificity of $89 \%$ in differentiating Alzheimer's disease with respect to controls [8]. The level of p-tau in CSF reflects the pathological process of neurofibrillary degeneration. The sensitivity of this biomarker for differentiating patients with $A D$ and the healthy population amounts to $74 \%$ and specificity $92 \%$ [8]. A high level of p-tau in the CSF seems to be diagnosed only in patients with Alzheimer's disease in comparison to the other dementia syndromes $[10,23,27,31,32,33$,] or an acute period of stroke [10].

The coexistence of biomarkers of $\beta$-amyloid concentration and neurofibrillary degeneration is crucial to increase the degree of certainty in diagnosing Alzheimer's disease. The tau/A $\beta_{1-42}$ ratio has a sensitivity of $89 \%$ and specificity of $90 \%$ [2]. The assessment of the ratio between p-tau and
$A \beta_{1-42}$ increases sensitivity (86\%) and specificity $(97 \%)$ of identification in relation to the healthy population as well as other types of dementia (sensitivity $80 \%$; specificity $73 \%$ ) [16]. Sensitivity and specificity of the tau/p-tau ratios amount to $96 \%$ and $100 \%$, respectively [2].

Numerous tests carried out to date have confirmed that patients without dementia with lower levels of $A \beta$ in the cerebrospinal fluid and higher levels of t-tau and $p$-tau represent a group at high risk of developing Alzheimer's disease dementia $[4,18,19,21,26]$.

There is no simple pathognomonic biomarker for $A D$, and the overall analysis of CSF biomarkers ( $A \beta$, t-tau, and $p$-tau) is valuable for diagnosis. The combination of existing biomarkers helps to differentiate the neurodegenerative processes; however, due to problems with each laboratory's specific norms, establishing the proper diagnosis could be challenging. Analysing only the raw concentrations of CSF is not sufficient for the diagnosis of the underlying pathology because existing discrepancies in protocols and storage of the CSF samples might result in a completely different outcome. Researchers suggest that a diagnosis-oriented interpretation of the CSF pattern is a better approach to support the diagnosis of AD [13].

The Erlangen Score Algorithm, proposed by Lewczuk et al. $[13,15]$ seems to be a partial solution of those inter-centre discrepancies. It is an easy-toimplement algorithm to interpret CSF biomarkers concentration simultaneously. It can be applied for routine diagnostic testing and to facilitate the interpretation of the results because it enables us to categorise the CSF results into several categories, reflecting different degrees and patterns of pathological scores instead of the simple dichotomy of normal and abnormal results.

The Erlangen Score Algorithm is cut-off-value-independent and divides subjects into five categories from 0 to 4 , in which 0 stands for the lack of the brain disease and 4 reflects probable AD.

See Table I for the interpretation details.

According to existing data, the Erlangen Score Algorithm is a useful tool to determinate the risk of developing $A D$ before the onset of dementia or to confirm the AD diagnosis. The Erlangen Score Algorithm allows simple and quick interpretation of the results and a risk assessment of dementia development in the course of $A D$ in patients with SCD and $\mathrm{MCl}$. 
Table I. The interpretation of the Erlangen Score Algorithm

\begin{tabular}{|lll|}
\hline Score & Interpretation & Pattern of biomarkers \\
\hline 0 & No evidence of organic CNS disease & Normal biomarkers \\
\hline 1 & AD improbable & Slightly altered results of either A $\beta$ or t-tau/p-tau, but not both \\
\hline 2 & AD possible & Clearly pathologic results of either A $\beta$ or t-tau/p-tau, but not both \\
\hline 2 & AD possible & Slight alteration of both A $\beta$ and $\mathrm{t}$-tau/p-tau \\
\hline 3 & AD possible & $\begin{array}{l}\text { Clearly pathologic results of either A } \beta \text { or t-tau/p-tau accompanied } \\
\text { by a slight alteration of the biomarker }(\mathrm{s}) \text { of the other group }\end{array}$ \\
\hline 4 & AD probable & All CSF AD biomarkers clearly pathologic \\
\hline
\end{tabular}

Table II. Participant characteristics for age, gender, and level of education in the three study groups

\begin{tabular}{|lcccc|}
\hline Variable & $\begin{array}{c}\text { Whole } \\
\text { sample }\end{array}$ & SCD & MCI & $\begin{array}{c}\text { AD- } \\
\text { dementia }\end{array}$ \\
\hline$n$ & 217 & 31 & 104 & 82 \\
\hline Age & $\begin{array}{l}65.62 \\
(9.84)\end{array}$ & $\begin{array}{c}59.23 \\
(7.68)\end{array}$ & $\begin{array}{c}63.8 \\
(9.23)\end{array}$ & $\begin{array}{c}70.34 \\
(9.25)\end{array}$ \\
\hline Gender, M/W & $89 / 128$ & $9 / 22$ & $45 / 59$ & $35 / 47$ \\
\hline Level of education & & & & \\
\hline Primary school & 34 & 1 & 14 & 19 \\
\hline Secondary school & 92 & 14 & 46 & 32 \\
\hline University & 91 & 16 & 44 & 31 \\
\hline
\end{tabular}

Data is presented as mean (standard deviation)

$M$ - men, $W$-women, SCD - subjective cognitive decline, $\mathrm{MCl}$ - mild cognitive impairment, $A D$ - Alzheimer's disease

The aim of this paper was to establish whether the analysis of the concentration of the CSF biomarkers with Erlangen Score Algorithm in subjects with $\mathrm{MCl}$ and SCD is useful in diagnosing and predicting the clinical progression to dementia due to Alzheimer's disease.

\section{Material and methods Participants}

A total of 217 patients (128 women and 89 men) were recruited between June 2011 and June 2014 in the Neurodegenerative Department of Neurology, Clinic of the Central Clinical Hospital in Warsaw, Poland. The study was approved by the Hospital Clinic's Ethics Committee. All subjects and/or their relatives gave their informed consent for the study.

The majority (58.9\%) of the 217 study participants were women. The average age was 65.62 (SD = 9.84) years. Thirty-four participants completed primary schools only, 92 had secondary school diplomas, and 91 had university degrees. Participants were recruited from three groups according to the clinical diagnosis.
In our study group, 31 persons were SCD subjects, 104 participants were diagnosed with $\mathrm{MCl}$, and 82 were patients with $A D$ dementia. The demographic characteristics are presented in Table II.

Within two days of hospitalisation, physicians conducted clinical interviews focusing on cognitive symptoms, coupled with physical, neurological, and psychiatric examinations with special emphasis on cognitive disorders. Screening cognitive tests and functional assessments were performed. Moreover, all the patients underwent neuropsychological evaluation. Furthermore, routine blood and brain imaging (magnetic resonance image [MRI] or computed tomography (T) were conducted. CSF from the lumbar puncture was obtained after all the above procedures.

The subjects were classified into three groups according to the clinical diagnosis. The patients with SCD $(n=31)$ were diagnosed according to Jessen et al.'s guidelines [12]. For $\mathrm{MCI}(n=104)$ we used Petersen et al.'s criteria [29]. Dementia due to AD ( $n=82$ ) was established by means of the recent criteria adopted by the National Institute on Aging - Alzheimer's Association and the European Federation of Neurological Societies [1].

Patients aged below 45 years, with brain tumours, severe depressive syndrome, previous diagnosis of a major psychiatric disorder, history of alcohol or drug abuse, and other severe medical conditions that might be causes of cognitive impairments were excluded from the study.

The average clinical follow-up was 14.33 months $(S D=6.82)$. To assess the progression of cognitive impairment, all non-demented subjects (SCD/MCI, $n=135$ ) were grouped together, and those who remained stable were described as SCD/MCI-S $(n=119)$, and participants whose results worsened were labelled as SCD/MCI-P (progressive, $n=16$ ). 
The second evaluation was based on neurological and neuropsychological assessments

\section{Cerebrospinal fluid analysis}

Six millilitres of CSF were obtained by means of a lumbar puncture with a non-traumatic spinal anaesthesia needle. After the lumbar puncture, only one patient reported a moderated headache. No other side-effects were registered. The CSF samples were centrifuged and stored in polypropylene tubes at $-80^{\circ} \mathrm{C} . \mathrm{A} \beta_{1-42}$, t-tau, and $\mathrm{p}$-tau concentrations were measured by using a sandwich enzyme-linked immunosorbent assay kit (ELISA) (Innogenetics, Gent, Belgium) in the hospital laboratory. The reference ranges were estimated in the hospital laboratory and were previously published elsewhere [17]. The cut-off values that were pathological for AD were as follows: $A \beta_{1-42}$ below $609.54 \mathrm{pg} / \mathrm{ml}$, t-tau above $277.02 \mathrm{pg} / \mathrm{ml}$, and $\mathrm{p}$-tau higher than $55.08 \mathrm{pg} / \mathrm{ml}$. To avoid a misdiagnosis in patients with the border scores, a 10\% border zone was also implemented.

\section{Results}

\section{Differences in the Alzheimer's disease biomarkers concentrations in the cerebrospinal fluid in the studied groups}

To assess the differences between levels of $A D$ biomarkers in CSF in all of the groups of patients we conducted one-way ANOVA. The results are shown in Table III.

We observed a significant main effect of diagnosis for all of the three biomarkers and two indexes. Patients with a more severe cognitive impairment had significantly lower levels of amyloid $A \beta_{1-42}, A \beta_{1-42} / t$-tau, $\mathrm{A} \beta_{1-42} / \mathrm{p}$-tau and significantly higher levels of the pro- teins t-tau and $p$-tau. $A \beta_{1-42} / p$-tau index accounted for the most pronounced differences between the groups.

The level of $A \beta_{1-42}$ differentiated participants in terms of diagnosis $\mathrm{F}(2,214)=36.29 ; p<0.001$; $\eta^{2}=0.25$. The SCD group had higher levels of $A \beta_{1-42}$ than those in the $\mathrm{MCl}$ group (LSD test, $M D=147.19$, $p=0.006$ ) and in the AD-dementia group (LSD test, $M D=404.43, p<0.001$ ), and those in the $\mathrm{MCl}$ group had higher levels than did the AD-dementia group (LSD test, $M D=257.25, p<0.001$ ).

The level of t-tau differentiated participants in terms of diagnosis $\mathrm{F}(2,214)=35.63 ; p<0.001$; $\eta^{2}=0.25$. The SCD group had lower levels of $t$-tau than those in the AD-dementia group (LSD test, $M D=-326.75, p<0.001$ ), and those in the $\mathrm{MCl}$ group had lower levels than did the AD-dementia group (LSD test, MD $=-288.16, p<0.001)$. There were no significant differences for the remaining groups.

The level of $p$-tau differentiated participants in terms of diagnosis $\mathrm{F}(2,214)=21.01 ; p<0.001$; $\eta^{2}=0.16$. The SCD group had lower levels of $p$-tau than those in the AD-dementia group (LSD test, $M D=-32.89, p<0.001)$, and those in the $M C l$ group had statistically lower levels than did the AD-dementia group (LSD test, MD = -29.31, $p<0.001$ ). There were no significant differences for the remaining groups.

The $A \beta_{1-42} / t$-tau ratio differentiated participants in terms of diagnosis $F(2,214)=37.65 ; p<0.001 ; \eta^{2}=0.26$. The SCD group had higher levels of $A \beta_{1-42} / t$-tau than those in the $\mathrm{MCl}$ group (LSD test, $M D=0.75$, $p=0.035$ ) and in the AD-dementia group (LSD test, $M D=2.62, p<0.001$ ), and those in the $\mathrm{MCl}$ group had higher levels than those in the AD-dementia group (LSD test, $M D=1.87, p<0.001$ ).

The $A \beta_{1-42} / p$-tau ratio differentiated participants in terms of diagnosis $F(2,214)=42.85 ; p<0.001$;

Table III. Differences in the Alzheimer's disease biomarker concentrations in the cerebrospinal fluid in different diagnosis statuses

\begin{tabular}{|lcccccc|}
\hline & SCD & MCI & AD-dementia & \multicolumn{3}{c|}{ ANOVA results } \\
\cline { 2 - 7 } & $M(S D)$ & $M(S D)$ & $M(S D)$ & $F$ & $p$ & $\eta^{2}$ \\
\hline$A \beta_{1-42}(\mathrm{pg} / \mathrm{ml})$ & $817.05(350.92)$ & $669.87(270.12)$ & $412.62(194.20)$ & 36.29 & $<0.001$ & 0.25 \\
\hline t-tau $(\mathrm{pg} / \mathrm{ml})$ & $277.61(166.48)$ & $316.20(200.98)$ & $604.36(326.43)$ & 35.63 & $<0.001$ & 0.25 \\
\hline $\mathrm{p}$-tau $(\mathrm{pg} / \mathrm{ml})$ & $46.77(19.36)$ & $50.35(25.94)$ & $79.66(43.99)$ & 21.01 & $<0.001$ & 0.16 \\
\hline $\mathrm{A} \beta_{1-42} / \mathrm{t}-\operatorname{tau}(\mathrm{pg} / \mathrm{ml})$ & $3.73(2.01)$ & $2.98(1.94)$ & $1.11(1.28)$ & 37.65 & $<0.001$ & 0.26 \\
\hline $\mathrm{A} \beta_{1-42} / \mathrm{p}$-tau $(\mathrm{pg} / \mathrm{ml})$ & $19.88(9.14)$ & $16.36(8.72)$ & $7.096 .41)$ & 42.85 & $<0.001$ & 0.29 \\
\hline
\end{tabular}

Data are presented as mean (standard deviation)

$A \beta_{1-42}$ - the CSF amyloid-A $\beta-42(\mathrm{pg} / \mathrm{ml}), t$-tau - the CSF total tau $(\mathrm{pg} / \mathrm{ml}), \mathrm{p}$-tau - the CSF hyperphosphorylated tau $(\mathrm{pg} / \mathrm{ml})$, SCD - subjective cognitive decline, $\mathrm{MCl}$ - mild cognitive impairment, $A D$ - Alzheimer's disease 
$\eta^{2}=0.29$. The SCD group had higher levels of $A \beta_{1-42} / p$-tau than those in the $M C l$ group (LSD test, $\mathrm{MD}=3.52, p=0.032)$ and in the AD-dementia group ( $L S D$ test, $M D=12.79, p<0.001$ ), and those in the $\mathrm{MCl}$ group had higher levels than those in the AD-dementia group (LSD test, $M D=9.27, p<0.001$ ).

\section{Cerebrospinal fluid abnormal levels of biomarkers in the studied group}

From the neurochemical perspective, the evaluation of biomarkers of Alzheimer's pathology in CSF in subjects with preclinical phases was helpful in the identification of persons who are particularly at the risk of developing dementia.

In patients with $S C D$, an abnormal, $A D$-specific level of $A \beta_{1-42}$ was found in $23.5 \%$ of patients, pathological levels of t-tau protein in were present in $31.8 \%$, and $p$-tau protein in $21.2 \%$ of participants. However, the pathological values of $A \beta_{1-42} / \mathrm{t}$-tau ratio were found in $20 \%$ of SCD patients, and $A \beta_{1-42} / p$-tau was present in $11 \%$ of them. It means that despite the absence of cognitive impairment in the neuropsychological assessment, individuals with subjective cognitive dysfunction present with typical $A D$ pathology, expressed by abnormal concentrations of neurochemical markers.
As expected, almost half of the patients with $\mathrm{MCl}$ diagnosis from the studied group had pathological levels of $A \beta_{1-42}(44.8 \%)$, t-tau protein (43.7\%), and p-tau protein (35.6\%). In contrast, the values of $A \beta_{1-42} / t$-tau ratio typical for $A D$ were found in $47.1 \%$ of patients with $\mathrm{MCl}$ and abnormal $\mathrm{A} \beta_{1-42} / \mathrm{p}$-tau ratio value was present in $39.1 \%$ of $\mathrm{MCl}$ subjects. It was also shown that in comparison to the SCD group, the abnormal concentrations of $A D$ biomarkers were more than twice as common in the $\mathrm{MCl}$ group, which is related to the much greater degree of cognitive impairment revealed in the neuropsychological assessment.

In the demented group, abnormal levels of $A \beta_{1-42}$ were observed in $91.3 \%$ of subjects, t-tau protein levels in $83.8 \%$ of them, and p-tau proteins in $75 \%$. Pathological values of $A \beta_{1-42} /$ t-tau ratio were found in $87.5 \%$ of patients with dementia along with the abnormal values of $A \beta_{1-42} / p$-tau ratio in $86.3 \%$ of participants with dementia due to AD. The distribution of abnormal CSF biomarkers values in the described group is shown in Table IV.

\section{Differences in the Erlangen Score in the studied groups}

The CSF biomarkers were analysed with respect to the Erlangen Score Algorithm. It was found that in

Table IV. Distribution of abnormal cerebrospinal fluid biomarkers values in study participants

\begin{tabular}{|lcccc|}
\hline Variable & $\begin{array}{c}\text { Whole sample } \\
(n=217)\end{array}$ & $\begin{array}{c}\text { SCD } \\
(n=31)\end{array}$ & $\begin{array}{c}\text { MCI } \\
(n=104)\end{array}$ & $\begin{array}{c}\text { AD-dementia } \\
(n=82)\end{array}$ \\
\hline $\mathrm{A} \beta_{1-42} \leq 609.54$ & $131(60.4 \%)$ & $10(32.3 \%)$ & $47(45.2 \%)$ & $74(90.2 \%)$ \\
\hline t-tau $\geq 277.02$ & $122(56.2 \%)$ & $10(32.3 \%)$ & $46(44.2 \%)$ & $66(80.5 \%)$ \\
\hline$p$-tau $\geq 55.08$ & $99(45.6 \%)$ & $9(29 \%)$ & $31(29.8 \%)$ & $59(72 \%)$ \\
\hline
\end{tabular}

$S C D$ - subjective cognitive decline, $M C l$ - mild cognitive impairment, $A D$ - Alzheimer's disease, $A \beta_{1-42}$-the CSF amyloid-A $\beta-42$ (pg/ml), $t$-tau - the CSF total tau $(\mathrm{pg} / \mathrm{ml})$, p-tau - the CSF hyperphosphorylated tau $(\mathrm{pg} / \mathrm{ml})$

Table V. The Erlangen Algorithm scores in three study groups

\begin{tabular}{|c|c|c|c|c|c|}
\hline $\begin{array}{l}\text { The Erlangen } \\
\text { Score }\end{array}$ & Risk of developing AD & $\begin{array}{l}\text { Whole sample } \\
\quad(n=217)\end{array}$ & $\begin{array}{c}\text { SCD } \\
(n=31)\end{array}$ & $\begin{array}{c}\mathrm{MCl} \\
(n=104)\end{array}$ & $\begin{array}{c}\text { AD-dementia } \\
\quad(n=82)\end{array}$ \\
\hline 0 & None & $52(24.0 \%)$ & 15 (48.4\%) & 33 (31.7\%) & $4(4.9 \%)$ \\
\hline 1 & Improbable & $6(2.8 \%)$ & 0 & $5(4.8 \%)$ & $1(1.2 \%)$ \\
\hline $2-3$ & Possible & $71(32.7 \%)$ & $13(41.9 \%)$ & $42(40.4 \%)$ & $16(19.5 \%)$ \\
\hline 4 & Probable & $88(40.6 \%)$ & $3(9.7 \%)$ & $24(23.1 \%)$ & $61(74.4 \%)$ \\
\hline $\begin{array}{l}\text { Underlying AD } \\
\text { pathology (2,3 and } \\
4 \text { scores together) }\end{array}$ & Possible and probable & $159(73.3 \%)$ & $16(51.6 \%)$ & $66(63.5 \%)$ & $77(93.9 \%)$ \\
\hline
\end{tabular}

Data presented in number of patients (percentage)

$S C D$ - subjective cognitive decline, $M C I$ - mild cognitive impairment, $A D$-Alzheimer's disease 
the group of the AD dementia patients, the result of the Erlangen Score Algorithm suggested an underlying $\mathrm{AD}$ pathology in $93.9 \%$ of the cases. In the $\mathrm{MCl}$ group, it amounted to $63.5 \%$ (in $23.1 \%$ of the participants defined as probable and in $40.4 \%$ as possible). Furthermore, in the SCD group, the Erlangen Score Algorithm results implied an underlying AD pathology in $51.6 \%$ of the cases $(9.7 \%$ as probable and $41.9 \%$ as possible). See Table $V$ for detailed data.

\section{Erlangen Score in the follow-up analysis}

We also analysed CSF biomarkers with respect to the Erlangen Score Algorithm after an average clinical follow-up of 14.33 months in subjects without dementia. Due to the relatively brief period of observation and small numbers of certain groups, progressive non-demented subjects (SCD/MCI-P) were analysed together. It transpired that in this progressive population (SCD/MCI-P) $(n=16)$ that 15 subjects scored two, three, or four points (93.75\%), confirming the AD pathology. Among the non-progressing subjects $(n=119)$ only $56.31 \%$ scored two, three, or four points, determining the risk of developing AD as possible (40.34\%) or probable (15.97\%). The higher the score, the greater the risk of progression of cognitive impairment $\left(\chi^{2}[3, n=135]=13.26\right.$; $p=0.004$, where $\mathrm{V}$ Kramer $=0.31)$. Table VI presents Erlangen Algorithm Scores for groups of progressive and non-progressive subjects.

\section{Discussion}

The characteristic profile of the biomarkers in CSF appears early and is maintained in the course of the development of Alzheimer's disease [19]. According to the criteria, the typical configuration of $A D$ biomarker in CSF confirms Alzheimer's disease as the reason behind cognitive disorders in patients with dementia [5,7,30]. The Erlangen Algorithm Scores ranging from 0 to 4 (Table I) enables not only the detection of the underlying pathology but also staging of the disease progression. Although the use of the Erlangen Algorithm Score is not worldwide, it seems to be a valuable tool for both diagnostics and prediction purposes. It also minimises the risk of misdiagnosis in clinically uncertain cases in patients with $\mathrm{MCl}$ and SCD. The differences between laboratories with respect to concentrations of the CSF biomarkers may occur from applying different techniques, reagents, methods of collecting and storing the cerebrospinal fluid, or statistical methods of evaluating the cut-off points for the subsequent parameters, specific to the laboratory [14]. The Erlangen Algorithm Score is cut-off-value-independent and can be easily adopted by laboratories irrespective of their analytical platform and the reference ranges. Therefore, it is suggested that the Erlangen Score Algorithm be implemented in the interpretation of the results, especially in situations when the subject provides the results from a facility with unknown laboratory norms.

The studied population (Table II) was divided into three groups ( $S C D, M C l$, and $A D$-dementia) regarding their level of impairment based on the clinical evaluation. Obtained results from the CSF biomarkers analysis pointed to the differences in the levels of $A \beta_{1-42}$, t-tau, and $p$-tau along with the levels of $A \beta_{1-42} / t$-tau and $A \beta_{1-42} / p$-tau ratios with respect to the subject's diagnosis (Table III). It allowed the categorisation of the CSF results, reflecting different degrees and constellations of pathological findings.

In our paper, we analysed the concentration levels of the CSF biomarkers ( $A \beta_{1-42}$; t-tau and $p$-tau) by means of the Erlangen Score Algorithm [15] in subjects with clinical diagnosis of SCD, $\mathrm{MCl}$, and AD-

Table VI. The Erlangen Algorithm Scores in follow-up analysis

\begin{tabular}{|c|c|c|c|c|}
\hline The Erlangen Score & Risk of developing AD & $\begin{array}{l}\mathrm{SCD} / \mathrm{MCl} \\
(n=135)\end{array}$ & $\begin{array}{l}\text { SCD/MCl-P } \\
(n=16)\end{array}$ & $\begin{array}{c}\text { SCD/MCl-S } \\
(n=119)\end{array}$ \\
\hline 0 & None & 48 (35.56\%) & $1(6.25 \%)$ & 47 (39.49\%) \\
\hline 1 & Improbable & $5(3.70 \%)$ & 0 & $5(4.20 \%)$ \\
\hline $2-3$ & Possible & $55(40.74 \%)$ & $7(43.75 \%)$ & $48(40.34 \%)$ \\
\hline 4 & Probable & $27(20.00 \%)$ & $8(50.00 \%)$ & $19(15.97 \%)$ \\
\hline $\begin{array}{l}\text { Underlying AD pathology 2, 3, } \\
\text { and } 4 \text { scores together }\end{array}$ & Possible and probable & $82(60.74 \%)$ & $15(93.75 \%)$ & $67(56.31 \%)$ \\
\hline
\end{tabular}

Data presented in number of patients (percentage)

$S C D$ - subjective cognitive decline, $\mathrm{MCl}$ - mild cognitive impairment, SCD/MCI-P-SCD/MCI progression, SCD/MCI-S-SCD/MCI stable 
dementia. The obtained results were in line with the existing data $[13,15]$, and in more advanced patients the highest Erlangen Score was the most common in $A D$ subjects, but the rarest in the SCD subjects. On the other hand, half of the clinically normal SCD population in our study had possible and probable $A D$ pathology, which makes this group more exposed for future risk of dementia development, despite the lack of any cognitive deficits observed in the detailed neuropsychological assessment. The $\mathrm{MCl}$ population was similar to the SCD subjects in terms of having a possible risk of $A D$, but the probable risk was increased, with a lower number of persons with no risk (Table IV). Because there are no published data of the Erlangen Score distribution in the non-demented population, our results are novel and those findings could shed some light on this area of research.

The utilisation of the Erlangen Algorithm Score enables for risk estimation of developing Alzheimer's-type dementia in patients with SCD and $\mathrm{MCl}$, long before the dementia symptoms are present. A higher Erlangen Score was related to an increased risk of conversion from $\mathrm{MCl}$ to $\mathrm{AD}$, and progression from SCD to $\mathrm{MCl}$. In our population, almost all of the observed progressive patients without dementia (15 of a total 16 progressive subjects) were characterised by probable and possible AD pathology (the Erlangen Score 2, 3, or 4), but only one patient form this subgroup had no (scored as 0 ) risk of developing AD. Similar results were presented in the work of Lewczuk et al. [13]. This is in accordance with the literature data and reflects the nature of development of Alzheimer's disease.

Our research has shown that an analysis of the concentrations of the CSF biomarkers by using the Erlangen Algorithm Score allows us to determine the likelihood of developing Alzheimer's disease in patients presenting subjective or mild cognitive impairment, even based on a relatively short period of observation. Those patients diagnosed with $\mathrm{MCl}$ and SCD who obtained 2-4 points during evaluation with Erlangen Algorithm Score require further observation because of the increased risk of $A D$ development. However, further observation is essential and the probability of conversion to dementia is increased because almost $13 \%$ (4 of 31 ) subjects with SCD progressed to $\mathrm{MCl}$ within the relatively short period of more than a year. In the more advanced $\mathrm{MCl}$ group, a similar percentage of patients (11.54\%, 12 persons) developed AD dementia after 14.33 months, which is similar to the results published by Lewczuk et al. (2015) [13]. In our combined group of non-demented progressive only one subject had no underlying Alzheimer's pathology, which confirms that the presence of possible or probable $A D$ in the Erlangen Algorithm Score increases the risk of $A D$ dementia, even in cognitively intact persons. Other data on the Erlangen Score values in the SCD subject progression to more advanced stages is unknown, which makes our results unique.

The Algorithm seems to be also more sensitive in detecting the conversion risk than using only neuroimaging techniques $[13,15,24]$. The combined hippocampus volumetric measures with AD-CSF biomarker concentrations could increase the specificity and the sensitivity of diagnosis [25], suggesting that $A \beta_{42}$ concentrations and hippocampal volumes may be used in combination to best identify prodromal $\mathrm{AD}[30]$. However, the most recent data suggest that the $A \beta$ level is superior to single biomarker levels or their combination [9].

As we expected, there were some limitations to the study. The subjects were recruited from the memory clinic, which made the results less representative for the whole population. Most of the patients had secondary or university level education. A high level of education of patients with a family history of $A D$ could be an additional factor to report to the memory clinic. The subsequent drawback was the lack of a healthy control group due to ethical reasons, as the procedure of a lumbar puncture is rather invasive. The relatively short period of observation is another disadvantage, but despite this, some results brought interesting conclusions and all subjects from the described group are under our medical control.

Current data suggest that the measuring the $A \beta_{42} / A \beta_{40}$ ratio makes the diagnosis of $A D$ more reliable, but it is very expensive and due to financial reasons, the additional use of the Erlangen Algorithm Score in the interpretation of the raw levels of CSF biomarkers might improve the diagnostic value.

At present, the limitations in applying the CSF biomarkers in daily practice are still related to the difficulties in interpreting the results. It is important to establish methods and protocols to improve early diagnosis of the AD. Determining the biomarkers identifying asymptomatic patients and estimating the risk of $A D$ developing will allow selection of individuals with $A D$ before the stage of dementia 
evolves. Patients with a high risk of developing $A D$ are the best group for further clinical research and clinical trials. Furthermore, it is of ethical importance because it is not desirable to include patients with a low risk of developing the disease into research accompanied by the risk of adverse effects. Identification of asymptomatic patients will also be of key importance in the future when causal treatment of Alzheimer's disease will be available

\section{Acknowledgments}

This study is part of the BIOMARKAPD project within the EU Joint Program for Neurodegenerative Disease Research and is supported through the National Centre for Research and Development (3/BIOMARKAPD/JPND/2012).

\section{Disclosure}

The authors report no conflict of interest.

\section{References}

1. Albert MS, DeKosky ST, Dickson D, Dubois B, Feldman HH, Fox NC, Gamst A, Holtzman DM, Jagust WI, Petersen RC, Snyder PJ, Carillo MC, Thies B, Phelps CH. The diagnosis of mild cognitive impairment due to Alzheimer's disease: recommendations from the National Institute on Aging - Alzheimer's Association workgroups on diagnostic guidelines for Alzheimer's disease. Alzheimers Dement 2011; 7: 270-279.

2. Avila JP, Lucas J. Role of tau protein in both physiological and pathological conditions. Physiol Rev 2004; 84: 361-384.

3. Blennow K. Cerebrospinal fluid protein biomarkers for Alzheimer's disease. NeuroRx 2004; 1: 213-225.

4. Blennow K, Vanmechelen E. CSF markers for pathogenic processes in Alzheimer's disease: diagnostic implications and use in clinical neurochemistry. Brain Res Bull 2003; 61: 235-342.

5. Clark CM, Xie S, Chittams J, Ewbank D, Peskind E, Galasko D, Morris JC, McKeel DW Jr, Farlow M, Weitlauf SL, Quinn J, Kaye J, Knopman D, Arai H, Doody RS, DeCarli C, Leight S, Lee VM, Trojanowski JQ. Cerebrospinal fluid tau and beta-amyloid: how well do these bio-markers reflect autopsy-confirmed dementia diagnoses? Arch Neurol 2003; 60: 1696-1702.

6. Fagan AM, Roe CM, Xiong C, Mintun MA, Morris JC, Holtzman DM. Cerebrospinal fluid tau/beta-amyloid(42) ratio as a prediction of cognitive decline in nondemented older adults. Arch Neurol 2007; 64: 343-349.

7. Fagan AM, Shaw LM, Xiong C, Vanderstichele H, Mintun MA, Trojanowski JQ, Coart E, Moriss JC, Holtzman DM. Comparison of analytical platforms for cerebrospinal fluid measures of beta-amyloid 1-42, total tau, and p-tau181 for identifying Alzheimer disease amyloid plaque pathology. Arch Neurol 2011; 68: 1137-1144.
8. Formichi P, Battisti C, Radi E, Federico A. Cerebrospinal fluid tau, A beta, and phosphorylated tau protein for the diagnosis of Alzheimer's disease. J Cell Physiol 2006; 208: 39-46.

9. Frölich L, Peters O, Lewczuk P, Gruber O, Teipel SJ, Gertz HJ, Jahn H, Jessen F, Kurz A, Luckhaus C, Hüll M, Pantel J, Reischies FM, Schröder J, Wagner M, Rienhoff O, Wolf S, Bauer C, Schuchhardt J, Heuser I, Rüther E, Henn F, Maier W, Wiltfang J, Kornhuber J. Incremental value of biomarker combinations to predict progression of mild cognitive impairment to Alzheimer's dementia. Alzheimers Res Ther 2017; 9: 84.

10. Hesse C, Rosengren L, Andreasen N, Davidsson P, Vanderstichele $\mathrm{H}$, Vanmechelen E, Blennow K. Transient increase in total tau but not phospho-tau in human cerebrospinal fluid after acute stroke. Neurosci Lett 2001; 297: 187-190.

11. Itoh N, Arai H, Urakami K, Ishiguro K, Ohno H, Hampel H, Buerger K, Wiltfang J, Otto M, Kretzschmar H, Moeller HJ, Imagawa M, Kohno H, Nakashima K, Kuzuhara S, Sasaki H, Imahori K. Largescale, multicenter study of cerebrospinal fluid tau protein phosphorylated at serine 199 for the antemortem diagnosis of Alzheimer's disease. Ann Neurol 2001; 50: 150-156.

12. Jessen F, Amariglio RE, van Boxtel M, Breteler M, Ceccaldi M, Chételat G, et al.; Subjective Cognitive Decline Initiative (SCD-I) Working Group. A conceptual framework for research on subjective cognitive decline in preclinical Alzheimer's disease. Alzheimers Dement 2014; 10: 844-852.

13. Lewczuk P, Kornhuber J, Toledo JB, Trojanowski JQ, KnapikCzajka M, Peters O, Wiltfang J, Shaw LM; US-ADNI. Validation of the Erlangen Score Algorithm for the prediction of the development of dementia due to Alzheimer's disease in pre-dementia subjects. J Alzheimers Dis 2015; 48: 433-441.

14. Lewczuk P, Kornhuber J, Wiltfang J. The German Competence Net Dementias: standard operating procedures for the neurochemical dementia diagnostics. J Neural Transm (Vienna) 2006; 113: 1075-1080.

15. Lewczuk P, Zimmermann R, Wiltfang J, Kornhuber J. Neurochemical dementia diagnostics: a simple algorithm for interpretation of the CSF biomarkers. I Neural Transm (Vienna) 2009; 116: 1163-1167.

16. Maddalena A, Papassotiropoulos A, Muller-Tillmanns B, Jung HH, Hegi T, Nitsch RM, Hock C. Biochemical diagnosis of Alzheimer disease by measuring the cerebrospinal fluid ratio of phosphorylated tau protein to beta-amyloid peptide42. Arch Neurol 2003; 60: 1202-1206.

17. Mandecka M, Budziszewska M, Barczak A, Pepłonska B, Chodakowska-Zebrowska M, Filipek-Gliszczynska A, Nesteruk M, Styczynska M, Barcikowska M, Gabryelewicz T. Association between cerebrospinal fluid biomarkers for Alzheimer's disease, APOE genotypes and auditory verbal learning task in subjective cognitive decline, mild cognitive impairment and Alzheimer's disease. J Alzheimers Dis 2016; 54: 157-168.

18. Mattsson N, Insel P, Nosheny R, Zetterberg H, Trojanowski JQ, Shaw LM, Tosun D, Weiner M; Alzheimer's Disease Neuroimaging Initiative. CSF protein biomarkers predicting longitudinal reduction of CSF beta-amyloid42 in cognitively healthy elders. Transl Psychiatry 2013; 3: e293.

19. Mattsson N, Zetterberg H, Hansson O, Andreasen N, Parnetti L, Jonsson M, Herukka SK, van der Flier WM, Blankenstein MA, 
Ewers M, Rich K, Kaiser E, Verbeek M, Tsolaki M, Mulugeta E, Rosen E, Aarsland D, Visser JP, Schroder J, Marcusson J, de Lon M, Hampel H, Scheltens P, Pritilla T, Walin A, Jonhagen ME, Minthon L, Winblad B, Blennow K. CSF biomarkers and incipient Alzheimer disease in patients with mild cognitive impairment. Am Med Assoc 2009; 302: 385-293.

20. Molinuevo JL, Blennow K, Dubois B, Engelborghs S, Lewczuk $P$, Perret-Liaudet A, Teunissen CE, Parnetti L. The clinical use of cerebrospinal fluid biomarker testing for Alzheimer's disease diagnosis: A consensus paper from the Alzheimer's biomarkers standardization initiative. Alzheimers Dement 2014; 10: 808-817.

21. Molinuevo JL, Gispert JD, Dubois B, Heneka MT, Lleo A, Engelborghs S, Pujol J, de Souza LC, Alcolea D, Jessen F. The AD-CSFIndex discriminates Alzheimer's disease patients from healthy controls: A validation study. J Alzheimers Dis 2013; 36: 67-77.

22. Morris JC. Early-stage and preclinical Alzheimer disease. Alzheimer Dis Assoc Disord 2005; 19: 163-165.

23. Nagga K, Gottfries J, Blennow K, Marcusson J. Cerebrospinal fluid phospho-tau, total tau and beta-amyloid (1-42) in the differentiation between Alzheimer's disease and vascular dementia. Dement Geriatr Cogn Disord 2002; 14: 183-190.

24. Nesteruk M, Nesteruk T, Styczyńska M, Barczak A, Mandecka M, Walecki J, Barcikowska-Kotowicz M. Predicting the conversion of mild cognitive impairment to Alzheimer's disease based on the volumetric measurements of the selected brain structures in magnetic resonance imaging. Neurol Neurochir Pol 2015; 49: 349-353.

25. Nesteruk M, Nesteruk T, Styczyńska M, Mandecka M, Barczak A, Barcikowska M. Combined use of biochemical and volumetric biomarkers to assess the risk of conversion of mild cognitive impairment to Alzheimer's disease. Folia Neuropathol 2016; 54: 369-374.

26. Parnetti L, Chiasserini D, Eusebi P, Giannandrea D, Bellomo G, De Carlo C, Padiglioni C, Mastrocola S, Lisetti V, Calabresi P. Performance of abeta1-40, abeta1-42, total tau, and phosphorylated tau as predictors of dementia in a cohort of patients with mild cognitive impairment. J Alzheimers Dis 2012; 29: 229-238.

27. Parnetti L, Lanari A, Amici S, Gallai V, Vanmechelen E, Hulstaert F. CSF phosphorylated tau is possible marker for discriminating Alzheimer's disease from dementia with Lewi bodies. Neurol Sci 2001: 22: 77-78.

28. Prestia A, Caroli A, Herholz K, Reiman E, Chen K, Jagust WJ, Frisoni GB. Translational Outpatient Memory Clinic Working Group; Alzheimer's Disease Neuroimaging Initiative. Diagnostic accuracy of markers for prodromal Alzheimer's disease in independent clinical series. Alzheimers Dement 2013; 9: 677-686.

29. Peteresen RC. Mild cognitive impairment as diagnostic entity. J Intern Med 2004; 256: 183-194.

30. Shaw LM, Vanderstichele H, Knapik-Czajka M, Clark CM, Aisen PS, Petersen RC, Blennow K, Soares H, Simon A, Lewczuk P, Dean R, Siemers E, Potter W, Lee VM, Trojanowski JQ. Cerebrospinal fluid biomarker signature in Alzheimer's disease neuroimaging initiative subjects. Ann Neurol 2009; 65: 403-413.

31. Sjögren M, Davidsson P, Tulberg M, Minthon L, Wallin A, Wikkelso C, Granérus AK, Vanderstichele H, Vanmechelen E, Blennow K. Both total and phosphorylated tau are increased in Alzheimer's disease. J Neurol Neurosurg Psychiatr 2001; 70: 624-630.
32. Sjögren M, Davidsson P, Wallin A, Granérus AK, Grundström E, Askmark H, Vanmechelen E, Blennow K. Decreased CSF-beta-amyloid 42 in Alzheimer's disease and amyotrophic lateral sclerosis may reflect mismetabolism of beta-amyloid induced by disparate mechanisms. Demen Geriatr Cogn Disord 2002; 13: 1112-1118.

33. Vanmechelen E, Vanderstichele H, Davidsson P, Van Kerschaver E, Van der Perre B, Sjogren M, Andreasen N, Blennow K. Quantification of tau phosphorylated at threonine 181 in human cerebrospinal fluid: a sandwich ELISA with a synthetic phosphopeptide for standardization. Neurosci Lett 2000; 285: 49-52. 